\title{
Gratingless enhanced microwave transmission through a subwavelength aperture in a thick metal plate
}

\author{
Alastair P. Hibbins ${ }^{a)}$ and J. Roy Sambles \\ Thin Film Photonics, School of Physics, University of Exeter, Stocker Road, Exeter, EX4 4QL, \\ United Kingdom \\ Chris. R. Lawrence \\ QinetiQ Ltd., Farnborough, GU14 OLX, United Kingdom
}

(Received 29 July 2002; accepted 14 October 2002)

\begin{abstract}
Remarkably enhanced transmission of microwave radiation through a single subwavelength slit in a thick metallic substrate surrounded by just a pair of parallel deep and narrow grooves is recorded. By also patterning the output face of the metal slab with two grooves there is strong exit beam confinement. There are no gratings in this structure and, hence, the transmission mechanism is not related to the conventional grating coupling of surface plasmons on the upper and lower surfaces of the substrate. Instead, the slit and the four grooves are all resonant, which is the essence of the functioning of the arrangement. The enhancement is due to the collective excitation of the FabryPérot mode in the slit and the cavity modes in the grooves. A finite-element modeling code is used to optimize the response of the structure, and to investigate the electromagnetic fields in the vicinity of the substrate. (C) 2002 American Institute of Physics. [DOI: 10.1063/1.1527704]
\end{abstract}

There is at present much interest in the remarkable transmission of subwavelength apertures in metal substrates. Since the work of Ebbessen and Lezec ${ }^{1}$ it has been reported that the extraordinary enhanced transmission of arrays of subwavelength apertures is associated with excitation and interaction of grating-coupled surface plasmon polaritons (SPPs) that propagate along both surfaces of the metal. ${ }^{2-4}$ More recently, Lezec et al. ${ }^{5}$ have proposed a similar idea to explain the enhanced transmission through a single aperture when the substrate faces are patterned periodically. Here, we present an experimental study of the enhanced transmission of electromagnetic radiation through a single slit. However, unlike the work discussed above, our samples are comprised of a narrow slit cut into a thick metallic substrate, and are positioned between just a single pair of deep and very narrow grooves. There is no grating, and as a consequence conventional grating-coupled SPPs on either metal surface cannot play a role in the mechanism responsible for the enhanced transmission.

A subwavelength slit in a thick metal plate may support a series of stationary, coupled surface modes, when its thickness is approximately equal to an integer number of half wavelengths (the Fabry-Pérot condition). ${ }^{6}$ At these wavelengths, the transmission of the structure is strongly enhanced. By adding two resonant grooves, one on either side of the slit on the illuminated face, it is shown that when modes in all three cavities are simultaneously excited and coupled together, the transmission efficiency is further increased by a factor of 3 . The transmitted radiation strongly diffracts when it emerges from the subwavelength slit, and the power is distributed uniformly over a cylindrical angle spread of $180^{\circ}$. However, this divergence is remarkably reduced by cutting a similar pair of resonant grooves into the

${ }^{a) E l e c t r o n i c ~ m a i l: ~ a . p . h i b b i n s @ e x e t e r . a c . u k ~}$ exit face. The mode in the slit will again couple to modes in the adjacent grooves, this time on the lower face of the substrate. The resulting three-source interference pattern produced by the three radiating cavities may, with a suitable choice of groove positioning, result in the structure resonantly "channeling" the power into a relatively nondivergent beam.

The sample manufactured for the present study is comprised of two pieces of aluminum-alloy sheet, of measured thickness $t_{s}=5.98 \pm 0.02 \mathrm{~mm}$ separated by the slit width $w_{s}$ $=0.26 \pm 0.03 \mathrm{~mm}$. A single groove (of depth $t_{g}=1.37$ $\pm 0.03 \mathrm{~mm}$ and width $w_{g}=0.26 \pm 0.02 \mathrm{~mm}$ ) is then cut into each surface of the two halves of the substrate, at a distance $d=4.41 \pm 0.03 \mathrm{~mm}$ from the slit center. The area of the sample $(250 \mathrm{~mm}$ by $250 \mathrm{~mm})$ is substantially larger than the

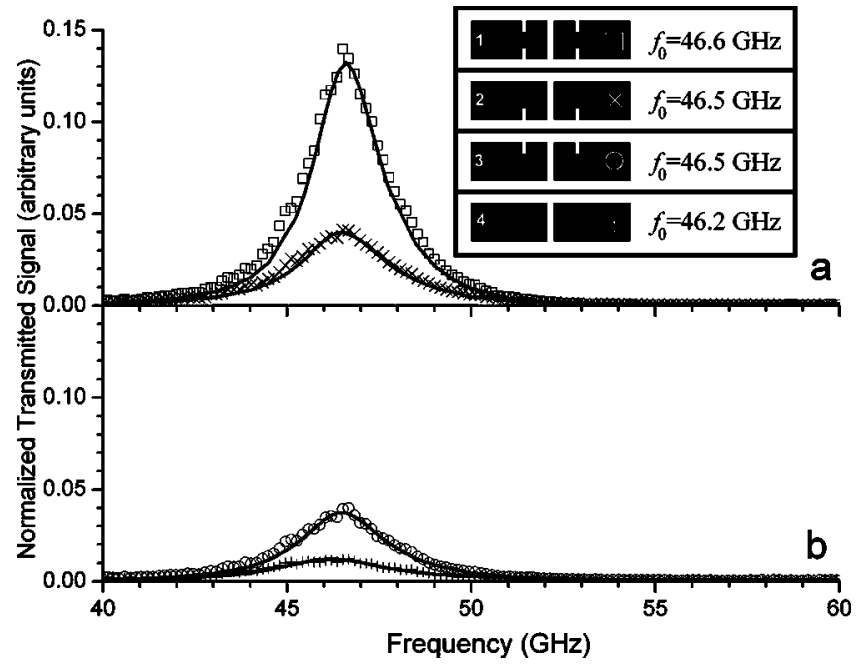

FIG. 1. Experimental normalized transmission spectra of the four samples studied. The resonant frequency of each sample is listed in the legend. The solid lines are the modeled transmissivities determined by fitting the predictions from the HFSS software to the experimental results. 
TABLE I. Parameters used in the HFSS modeling to produce the best fits to the experimental data (Fig. 1), where $d^{\prime}=d-\frac{1}{2}\left(w_{s}+w_{g}\right)$.

\begin{tabular}{lccccc}
\hline \hline \multicolumn{1}{c}{ Grooves } & $w_{s}(\mathrm{~mm})$ & $w_{g}(\mathrm{~mm})$ & $t_{s}(\mathrm{~mm})$ & $t_{g}(\mathrm{~mm})$ & $d^{\prime}(\mathrm{mm})$ \\
\hline 1. Upper and lower face & 0.255 & 0.260 & 5.96 & 1.33 & 4.15 \\
2. Lower face & 0.260 & 0.260 & 5.97 & 1.33 & 4.15 \\
3. Upper face & 0.260 & 0.260 & 5.97 & 1.33 & 4.15 \\
4. None & 0.260 & $\cdots$ & 6.00 & $\cdots$ & $\cdots$ \\
\hline \hline
\end{tabular}

diameter of the incident microwave beam $(\approx 30 \mathrm{~mm})$, and hence, the illuminated surface is effectively infinite in extent. The microwave source (which is comprised of a HP83556A mm-wave source module, HP83550 series rf plug in, and HP8350 series sweep oscillator) generates radiation of wavelength $\quad 5.0<\lambda_{0}<7.5 \mathrm{~mm} \quad(40<f<60 \mathrm{GHz}, \quad$ error $\pm 90 \mathrm{MHz}$ ), which is linearly polarized with its electric-field vector in the plane of the surface, perpendicular to the groove direction. Radiation from a horn antenna is normally incident upon the sample $\left(\theta=0^{\circ}\right)$. A second horn antenna is attached to an arm that extends from the center of a computer-controlled rotating table above which the slit of the sample is positioned. The transmissivity of the structure is then measured as a function of frequency and transmission angle $(\theta)$.

In order to understand and predict the electromagnetic response of the samples studied we have used the highfrequency structure simulator (HFSS) computer-modeling software $^{7}$ that is based on the finite-element method. All the modeling undertaken in this letter mirrors the experiment by assuming a normally incident plane wave (width, $30 \mathrm{~mm}$ ) centered upon the slit.

Figure 1(a) illustrates the experimentally derived transmission spectra of the sample discussed above $(\square$; sample 1). The data have been recorded with the signal horn antenna (of width $20 \mathrm{~mm}$ ) and detector positioned at a perpendicular distance of $90 \mathrm{~mm}$ from the sample $\left(\theta=180^{\circ}\right)$, and orientated so that it only detects radiation polarized with its $E$ vector in the plane of the surface, perpendicular to the groove direction. (None of the samples studied exhibit polarization conversion.) Also shown in Fig. 1(a) are the corresponding data for a sample that is manufactured without grooves on the illuminated side ( $\times$; sample 2$)$. Figure $1(b)$ illustrates the response of two further similar samples, one with grooves only on the illuminated face $(\bigcirc$; sample 3$)$, and the other without grooves on either face (+; sample 4). The solid lines are the respective best-fit transmissivities obtained by allowing the geometric parameters in the HFSS model to vary slightly from their measured values and comparing the predictions to the experimental results. Table I lists the pa-

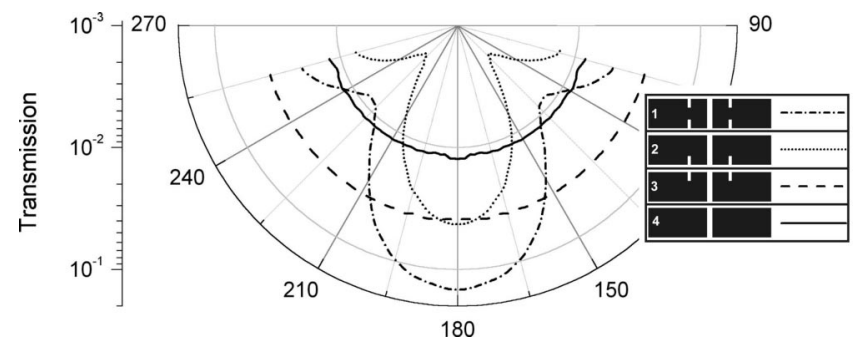

FIG. 2. Experimental beam directivity measured at the resonant frequency

mately 20 times greater than the incident field).
of each of the samples. rameter sets used, and they agree well with the measured values. However, it is evident from Fig. 1 that the samples do not all resonantly transmit at exactly the same frequency. This is primarily attributed to slight variations between the samples with respect to the thickness of the metal plates.

The directionality of the transmitted beam for each sample is measured at its resonant frequency (Fig. 2). Clearly, in the absence of grooves, the beam will radiate uniformly in all directions. The sample with grooves only on the illuminated face (dashed line) will also behave in a similar way; however, the two samples with grooves on the lower face show a strongly angle-dependent response. It is clear that while grooves on the illuminated (upper) face enhance the transmission by improving the coupling-in efficiency, grooves on the lower face give directionality to the beam.

In order to help further our understanding of the enhanced transmission and directivity phenomena, the HFSS software has been used to examine the electromagnetic fields on resonance of the structure. Figures 3 and 4 illustrate the time-averaged electric-field strength, magnetic-field strength, and Poynting vector distribution close to the substrate of sample 1 with radiation incident from above. It is clear from Fig. 3 that the slit exhibits $n=2$ Fabry-Pérot-like resonance, with regions of high-field strength at either end, and at its midlength. Hence, a narrow slit should transmit radiation of frequency, $n c / 2 t_{s} \approx 50 \mathrm{GHz}$, however, as predicted by Takakura $^{6}$ (and confirmed by our studies), the resonance is shifted to a lower frequency $(\sim 46 \mathrm{GHz})$ due to the slit's finite width. While optimizing the throughput of the system using the HFSS model, it becomes evident that the transmission efficiency is strongly dependent on the geometry and position of the grooves on the illuminated surface. This is because transmission efficiencies in excess of those obtainable through a single slit are only achieved when radiation of the same frequency is also able to resonantly couple to cavity modes in the upper pair of grooves. It is the coupling to-

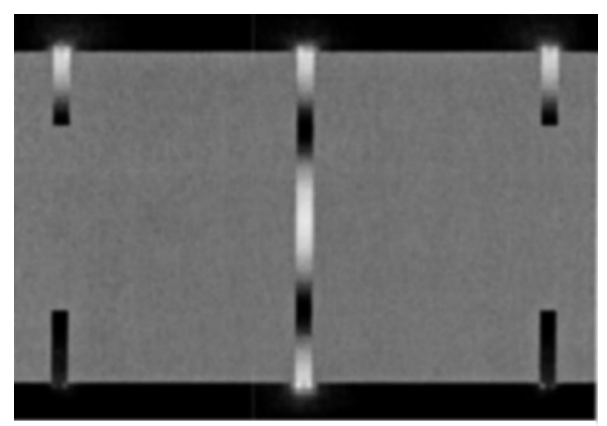

FIG. 3. Predicted time-averaged electric-field strength (sample 1). Radiation is incident from above at the resonant frequency of the slit. The gray scale is linear with white areas corresponding to most enhanced fields (approximately 20 times greater than the incident field). 


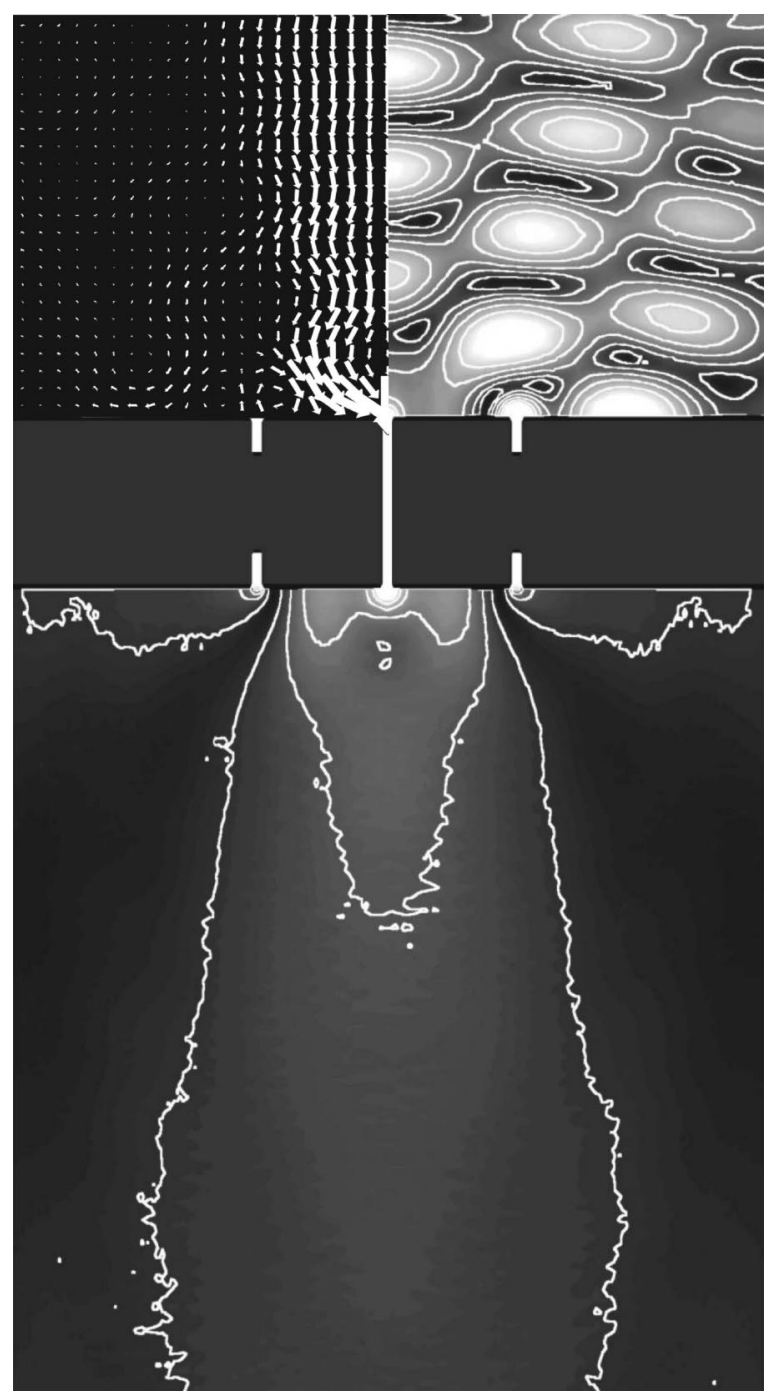

FIG. 4. Predicted electromagnetic fields for sample 1 with plane-wave radiation incident normally from above, at the resonant frequency $(46.6 \mathrm{GHz})$ of the slit. Above the substrate, the Poynting vector distribution $(\underline{S})$ is illustrated on the left of the mirror symmetry line, where the length and width of the arrows scale linearly with the magnitude of $\underline{S}$. The time-averaged magnetic-field strength $(H)$ is plotted as a gray scale, to the right of the symmetry line and also below the substrate. The gray scale is linear with white areas corresponding to field enhancements at least twice that of the incident field.

gether of these three modes that is responsible for the extraordinary results. In order to illustrate this phenomenon, the total transmissivity of the structure (integrated over $180^{\circ}$ ) has been modeled at the resonant frequency of the slit as a function of the depth of the upper grooves (Fig. 5). The two peaks in Fig. 5 correspond to groove depths at which the first (Fig. 3) and second-order cavity modes are excited.

The grooves on the lower surface have no significant effect on the total integrated power-they perform a different role. The mode in the slit will couple energy to the modes in the lower grooves, which will in turn reradiate power. Hence, the position of the grooves can be optimized so that the three "sources" produce an interference pattern that results in a strong lobe in the forward direction. This effect is clearly visible in the experimental results (Fig. 2) and also in the modeled magnetic field (Fig. 4). Not surprisingly, the opti-

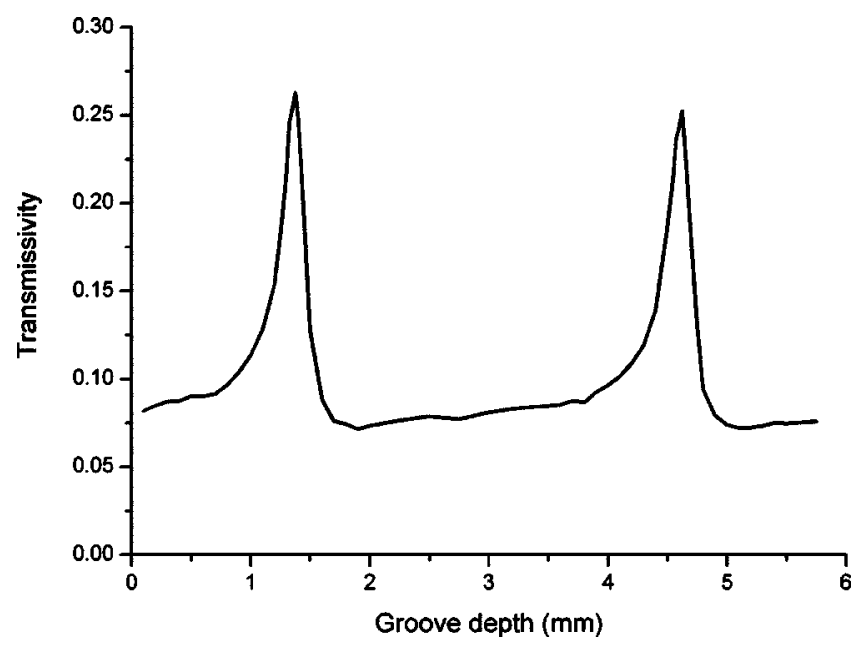

FIG. 5. Predicted total transmissivity (integrated over $180^{\circ}$ ) of a structure with grooves on the illuminated surface only. In these calculations, $w_{s}$ $=w_{g}=0.26 \mathrm{~mm}, t_{s}=6.00 \mathrm{~mm}$, and $d^{\prime}=4.15 \mathrm{~mm}(d=4.41 \mathrm{~mm})$ with incident radiation of frequency $46.2 \mathrm{GHz}$.

mum condition to out-couple radiation from the slit in order to give as near a plane-wave output as possible is also the most efficient geometry to couple in an incident plane wave.

To summarize, we have presented a study of the enhanced transmission of radiation through a simple slit in a thick metal substrate when the slit is positioned at the center of a pair of grooves. These remarkable results are attributed to the interaction of the Fabry-Pérot-like resonances in the slit coupling to similar cavity modes in the adjacent cavities. It has been demonstrated that while grooves on the illuminated surface may increase the total power flow through the slit, grooves on the lower surface of the substrate may be used to restrict the directionality of the beam to a limited angle range.

Such a structure is a generic type of microwave "lens." It opens up potential for reshaping microwave beams, providing enhanced transmission through opaque metal plates, forming line sources of high-brightness microwaves, producing line sources of low-beam divergence, etc. The essentials of the idea may also be used at infrared and even visible wavelengths. In addition, since the emission profile is not diffraction limited by the narrow slit, it is expected to have uses in the communication and nanolithography industries. Its application as a collecting mechanism for a microwave or optical detection system should also be noted.

This work was carried out as part of Technology Group 09 of the MoD Corporate Research program.

${ }^{1}$ T. W. Ebbesen and H. J. Lezec, Nature (London) 391, 667 (1998).

${ }^{2}$ E. Popov, M. Nevière, S. Enoch, and R. Reinisch, Phys. Rev. B 62, 16100 (2000).

${ }^{3}$ L. Martin-Moreno, F. J. Garcia-Vidal, H. J. Lezec, K. M. Pellerin, T. Thio, J. B. Pendry, and T. W. Ebbesen, Phys. Rev. Lett. 86, 1114 (2001).

${ }^{4}$ A. Krishnan, T. Thio, T. J. Kim, H. J. Lezec, T. W. Ebbesen, P. A. Wolff, J. Pendry, L. Martin-Moreno, and F. J. Garcia-Vidal, Opt. Commun. 200, 1 (2001)

${ }^{5}$ H. J. Lezec, A. Degiron, E. Devaux, R. A. Linke, L. Martin-Moreno, F. J. Garcia-Vidal, and T. W. Ebbesen, Science 297, 820 (2002).

${ }^{6}$ Y. Takakura, Phys. Rev. Lett. 86, 5601 (2001).

${ }^{7}$ Ansoft Corporation, Pittsburgh, PA. 